\title{
Towards an Understanding of Prompt GRB Emission.
}

\author{
Nicole M. Lloyd-Ronning
}

Canadian Institute for Theoretical Astrophysics

\begin{abstract}
We discuss the prompt emission of Gamma-Ray Bursts in different spectral energy bands. First, we suggest that a three-part synchrotron emission model [1,2] is a good description of the $\sim 20 \mathrm{keV}-1 \mathrm{MeV}$ gamma-ray emission of GRBs. We show that this model provides excellent fits to the data and naturally explains the observed global correlations between spectral parameters. In particular, we show there exists a negative correlation between between the peak of the $v F_{v}$ spectrum, $E_{p}$, and the low energy photon index $\alpha$ for bursts with $-2 / 3<\alpha<0$, and suggest that this correlation is due to the mechanism responsible for producing $\alpha$ 's above the value of $-2 / 3$ - namely, a decreasing mean pitch angle of the electrons. We then discuss the physical origin of the increasing number of GRBs that are observed to peak in the X-ray energy band $(\sim 5-40 \mathrm{keV})$. Although either a cosmological (i.e. high redshift) or intrinsic interpretation for the low values of $E_{p}$ is viable at this point, the data appear to suggest that intrinsic effects are playing the dominant role. Finally, we briefly comment on the prompt GRB optical emission $(\sim \mathrm{eV})$ and very high energy emission $(>10 \mathrm{MeV})$, and how these spectral bands may be used to place additional constraints on the physics of gamma-ray bursts.
\end{abstract}

\section{INTRODUCTION}

Just as broadband observations were (and are) necessary to establish the general external shock paradigm of the GRB afterglow, so are broadband data necessary to fully understand the physics of the prompt GRB emission. The prompt ( $\sim$ first one hundred seconds or so) emission gives us a unique opportunity to explore the physics of internal - and possibly external - shocks, address fundamental issues associated with the behavior of relativistic plasmas, particle acceleration and turbulence, and possibly even gain insight into the GRB progenitor itself. In this paper, I will present some recent advancements in our understanding of GRB prompt emission, and discuss important remaining questions associated with the initial phases of a burst.

\section{PROMPT GAMMA-RAY EMISSION}

Most GRB spectra in the energy range $(\sim 20 \mathrm{keV}-\sim$ $\mathrm{MeV}$ ) are well described by a so-called Band spectrum [3]. This model is essentially a smoothly broken power with a low energy photon spectral index $\alpha$, a high energy photon index $\beta$, and a break energy $E_{p}$. There have been some attempts to explain or interpret the global properties of these spectral parameters in terms of a physical model $[4,5,6]$, with inconclusive results. In particular, it was thought from very early on that the radiation mechanism responsible for GRBs (in this energy range) is synchrotron emission [7]. However, problems with this model - particularly with the behavior of the low energy $\gamma$-ray spectrum - were quickly brought to light. First, it was noted [5] that a number of bursts have a low energy spectral index $\alpha$ that falls above the so-called "line-ofdeath" value of $-2 / 3$, the asymptotic limit of an instantaneous (i.e. non-cooling) optically thin synchrotron spectrum from an isotropic, power-law electron distribution with some minimum cutoff energy. Even more fatal for the standard synchrotron picture, it was pointed out [6] that if particles are injected at once and then left to radiate, the synchrotron cooling times are very short (much less than the detector integration time). In this case, all of the particles cool and the electron spectrum becomes a soft power law (of index -2) at low energies. As a result, the asymptotic (upper) limit of the low energy photon spectrum is $\alpha=-3 / 2$. Nearly all GRBs have observed values of $\alpha$ that fall above this limit! However, as we will show below, when some of the simplifying assumptions in these models are modified, synchrotron emission in fact does a very good job of explaining the observed data.

\section{A Three-part Synchrotron Model}

Our model (described in more detail in [1] and [2]) modifies the usual simple picture of optically thin syn- 


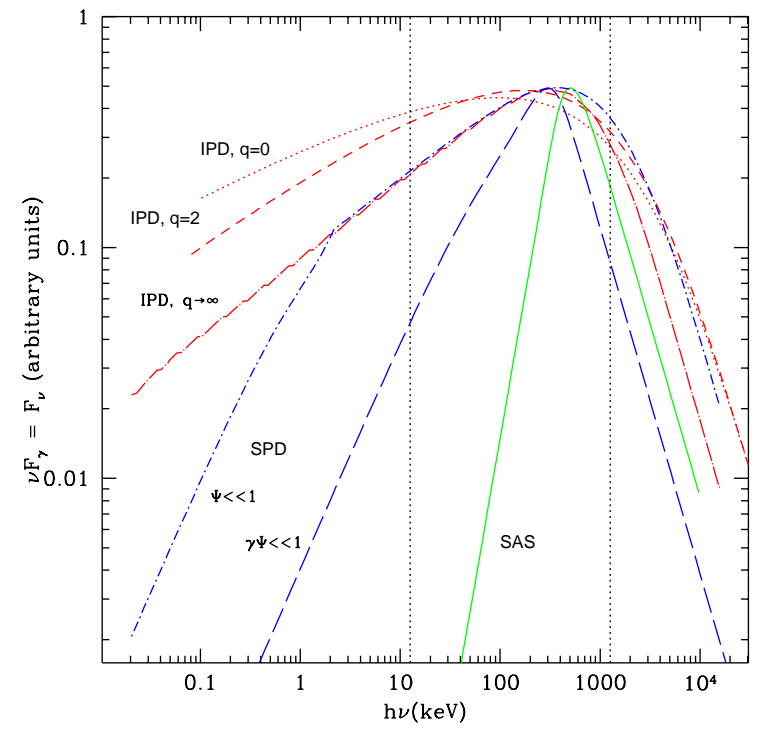

FIGURE 1. Synchrotron spectra in the various emission regimes (see text for explanation). The dotted vertical lines represent the approximate width of the BATSE spectral window.

chrotron emission from a power law distribution of electrons with a sharp low energy cutoff, by accounting for: 1) the possibility of a smooth cutoff to the low energy electron distribution, 2) radiation from an anisotropic electron distribution with a small mean pitch angle, 3) synchrotron self-absorption, and 4) the important instrumental effect in which the value of the fitted parameter $\alpha$ decreases as $E_{p}$ approaches the lower edge of the BATSE window. We have envisioned a realistic scenario in which particle acceleration and synchrotron losses occur continually and simultaneously behind each internal shock, with the characteristic acceleration time shorter than the loss time; this means that synchrotron loss effects are only evident in the particle distribution spectrum at energies much larger than what is relevant for our discussion here (at energies where the inequality is reversed and the loss time becomes shorter than the acceleration time). The emission in this model can be characterized by three distinct regimes.

1) IPD. This is the familiar optically thin synchrotron emission from a power law electron energy spectrum, with an isotropic pitch angle distribution. In contrast to most analyses, however, here we consider an electron distribution with a smooth low energy cutoff: $N(\gamma) \propto$ $\frac{\left(\gamma / \gamma_{m}\right)^{q}}{1+\left(\gamma / \gamma_{m}\right)^{p+q}}$. Note that for high energies $\left(\gamma>\gamma_{m}\right)$, the spectrum goes as $\gamma^{-p}$, while for low energies $\left(\gamma<\gamma_{m}\right)$, the spectrum goes as $\gamma^{q}$. Hence, $q$ denotes the steepness of the electron low energy cutoff (note that an actual "cutoff", in the sense that $N(\gamma) \rightarrow 0$ as $\gamma \rightarrow 0$, requires $q>0$ ). The asymptotic behavior of the synchrotron (photon number) spectrum for $q>-1 / 3$ is:

$$
F_{\gamma}= \begin{cases}v^{-2 / 3} & v \ll v_{m}=\frac{2}{3} v_{B} \sin \Psi \gamma_{m}^{2} \\ v^{-(p+1) / 2} & v \gg v_{m}\end{cases}
$$

where $F_{\gamma}$ is the photon flux, $\Psi$ is the electron pitch angle, and $v_{B}=\frac{e B}{m_{e} c}$ where $B$ is the magnetic field. Note that the peak of the $v F_{v}$ spectrum will occur at $E_{p} \propto v_{m} \propto$ $B \sin \Psi \gamma_{m}^{2}$, and that the aymptotic low energy index below this break is $\alpha=-2 / 3$.

In this regime, we expect a positive correlation between $E_{p}$ and $\alpha$ due to instrumental effects alone. If $E_{p}$ is close to the edge of the BATSE window, the low energy photon index may not yet have reached its asymptotic value and a smaller (or softer) value of $\alpha$ (relative to the asymptotic value) will be determined. A smooth cutoff to the electron energy distribution will exacerbate this effect because for a smoother cutoff (or a smaller value of $q$ ), the asymptote is reached at lower energies relative to $E_{p}$. Note that a dispersion in the smoothness of the low energy cutoff will tend to wash this correlation out to some degree, as seen in Figure 4 of [1]. For the cases of small pitch angle radiation and the self-absorbed spectrum (see below), this instrumental effect will be weaker because the low energy asymptotes are reached more quickly (i.e. at energies closer to $E_{p}$ ) than for the isotropic optically thin case (see Figure 1).

2) SPD. This type of synchrotron spectrum results from optically thin synchrotron emission by electrons with a mean pitch angle $\Psi \ll 1$; the analysis of synchrotron radiation in this regime was first done by [8]. For low density, high magnetic field plasmas expected in GRBs, the Alfvén phase velocity is greater than the speed of light and (therefore) the speed of the relativistic particles under consideration here. In this case, the pitch angle diffusion rate of the electrons interacting with plasma turbulence is smaller than the acceleration rate; consequently, the accelerated electrons could maintain a highly anisotropic distribution as required in the small pitch angle model. The shape of this spectrum depends on just how small the pitch angle is. For $\Psi \ll 1$, but $\Psi \gamma_{m} \sim 1$, we have:

$$
F_{\gamma}= \begin{cases}v^{0} & v \ll v_{s}=\frac{2}{3} v_{B} /\left(\gamma_{m} \Psi^{2}\right) \\ v^{-2 / 3} & v_{s} \ll v \ll v_{m} \\ v^{-(p+1) / 2} & v_{m} \gg v\end{cases}
$$

There are two breaks in this spectrum - one at $v_{m}$ and one at $v_{s}$. Because the Band spectrum can only accommodate one break, spectral fits to this model will put the parameter $E_{p}$ at one or the other of these two breaks, but most likely at $v_{m}$ because for $p>5 / 3$ (or for high energy photon index $\beta<-4 / 3$ which is the case for most bursts), the break across $v_{m}$ is more pronounced than across $v_{s}$. In 
this case, the low energy photon index $\alpha$ will fall somewhere between $-2 / 3$ and 0 .

However, as the pitch angle $\Psi$ decreases such that $\Psi \ll 1 / \gamma_{m}$, then the $v^{-2 / 3}$ portion of the spectrum disappears, and only the $v^{0}$ portion is left. In this case we have:

$$
F_{\gamma}= \begin{cases}v^{0} & v \ll v_{s}=\frac{4}{3} v_{B} \gamma_{m} \\ v^{-(p+1) / 2} & v_{s} \gg v,\end{cases}
$$

where $E_{p} \propto B \gamma_{m}$ (see [8] for a more detailed description of the behavior of the spectrum in this regime).

Here, we expect evidence of a negative correlation between $E_{p}$ and $\alpha$ as we transition from the IPD to the SPD regime, i.e. for $-2 / 3<\alpha<0$. In this case, the pitch angle decreases so that $E_{p} \propto \sin \Psi$ decreases, if all other physical parameters $\left(B\right.$ and $\left.\gamma_{m}\right)$ remain constant. In addition, as we go from the small pitch angle regime, $\Psi \gamma_{m} \sim 1(\Psi \ll 1)$, to the very small pitch angle regime, to $\Psi \gamma_{m} \ll 1$, the $v^{-2 / 3}$ portion of the spectrum disappears, and we are left with only the $v^{0}$ portion. In other words, as the mean of the pitch angle distribution decreases to very small values, $E_{p}$ decreases and the value of $\alpha$ decreases from $-2 / 3$ to 0 . This negative correlation will compete with the positive instrumental correlation mentioned above.

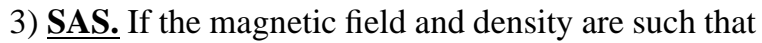
the medium becomes optically thick to the synchrotron photons with frequency $v<v_{a}$, then, for $v_{a}<v_{m}$, we have the following spectrum:

$$
F_{\gamma}= \begin{cases}v^{1} & v \ll v_{a}, \\ v^{-2 / 3} & v_{a} \ll v \ll v_{m}, \\ v^{-(p+1) / 2} & v_{m} \gg v\end{cases}
$$

In that case, $E_{p} \propto v_{a} \sim 10(n l)^{3 / 5} B^{2 / 5} \gamma_{m}^{-8 / 5} \Gamma^{9 / 5} \mathrm{~Hz}$, where $l$ and $n$ are the path length and particle density in the co-moving frame, and we have assumed an electron energy distribution index $p=2$. For $v_{a}>v_{m}$ we just have one break at $v_{a}$ with a low energy photon index of $\alpha=3 / 2$ (in both the isotropic and small pitch angle cases). The possibility of self-absorption in GRBs is a controversial issue. We have shown [1] that there are bursts for which a self-absorbed spectrum is a better fit than an optically thin one. We also found that in these cases, the absorption frequency tends to be near the lower edge of the BATSE window. In addition to this, Strohmeyer et al. [9] found that a number of bursts observed by GINGA with $E_{p}$ 's in the range 2 to $100 \mathrm{keV}$ have steep $(\alpha \sim 1)$ low energy spectral indices consistent with a self-absorbed spectrum. This raises interesting questions about the physics of the ambient plasma, because self-absorption in a GRB requires fairly large $\left(\sim 10^{8} G\right)$ magnetic fields and particle densities $\left(\sim 10^{8} \mathrm{~cm}^{-3}\right)$. The physical processes required to
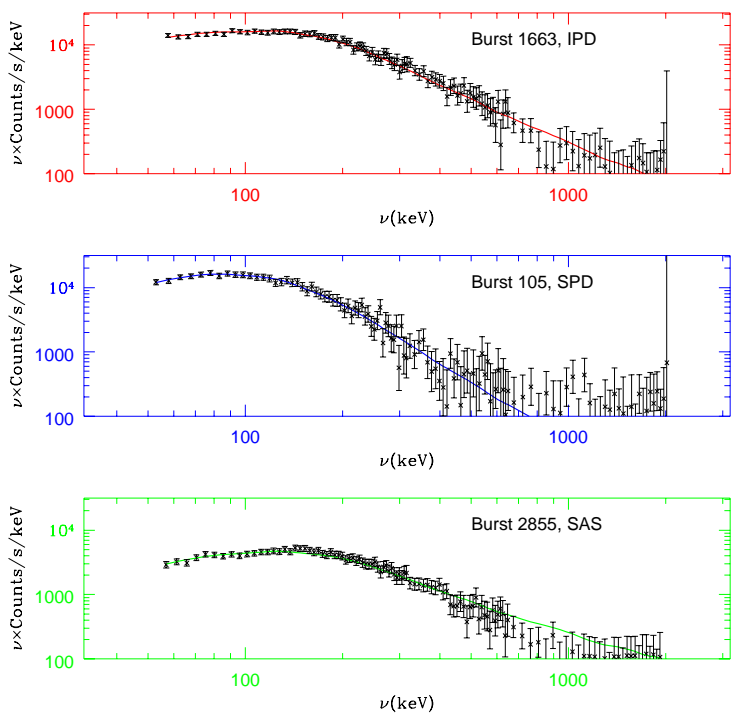

FIGURE 2. Three time resolved spectra fit to our generalized synchrotron model. The best fit emission regime corresponds to what is predicted by the low energy Band parameter $\alpha$.

achieve these conditions will need to be theoretically established if the data prove self-absorption to be a viable model.

\section{How the Data Stand Up}

Spectral Fits: We fit each spectrum to all 3 emission scenarios and then evaluate the fits based on their values of a reduced $\chi^{2}$. In Figure 2, we show examples of spectral fits in each emission regime. Each fit is taken at a time during the burst spectral evolution when the $\alpha$ parameter corresponded to the respective model. For example, in the top panel - burst 1663 - the spectrum is from a time when $\alpha \sim-2 / 3$, while in the middle panel - burst 105 - the spectrum is from a time in the profile when $\alpha=0$. Similarly, for the bottom panel, this spectrum corresponds to a time when $\alpha=1$. The reduced $\chi^{2}$ are 0.34 , 0.33 , and 0.50 for the top, middle and bottom panels respectively. In general the best model turns out to correspond to the emission regime suggested by Band's $\alpha$ values, which confirms our proposed method of physically interpretating Band fits based on the bursts' low energy photon index (for example, an IPD fit to the spectrum of burst 105 gave a $\chi^{2}>1$ compared to the $\chi^{2}=0.33$ for an SPD fit).

Global $\alpha$ Distribution: As discussed above, the low energy photon index $\alpha$ is the best parameter for distinguishing between the various synchrotron regimes. Figure 3 shows a histogram of $\alpha$ (taken from 2,026 time 


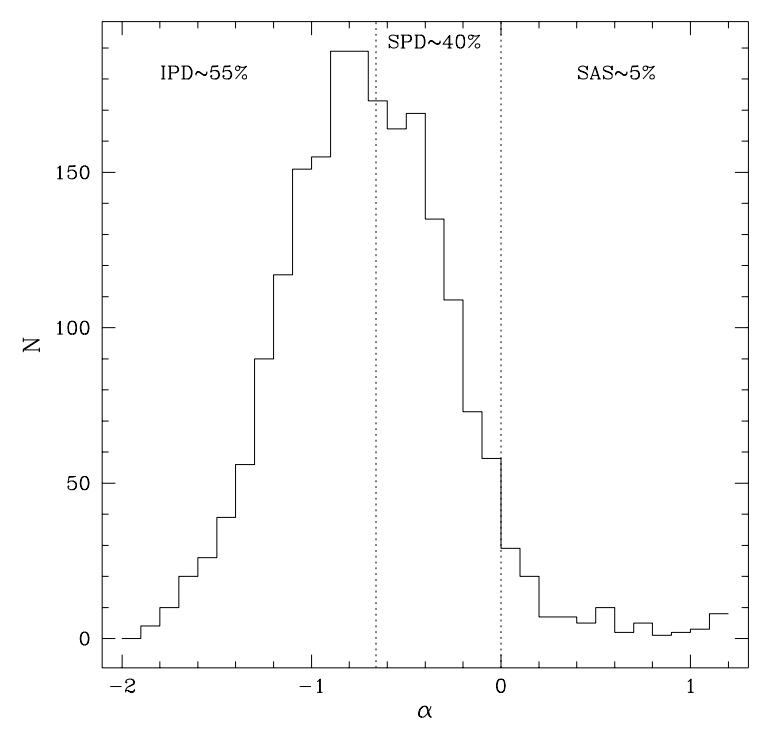

FIGURE 3. Histogram of the low energy spectral index $\alpha$, with each emission regime delineated. The data are from Preece et al., 1999.

resolved spectra with Band spectral fits, from [11]) with each regime clearly marked. Note that there are a significant number of spectra in the SPD regime.

Although the error bars on $\alpha$ can make some difference in the numbers of spectra in each regime, this does not affect the qualitative nature of our conclusions below (see [2] for a more detailed discussion on how the error on $\alpha$ affects this distribution). We now discuss the correlations present in the data and their consistency with what we expect in the context of the three synchrotron emission scenarios.

Observed Correlations Figure 4 shows the binned average correlation between $E_{p}$ and $\alpha$ present in $\sim 2000$ time resolved spectra from [11]. We have sorted $\alpha$ in ascending order and binned the data every 100 points (the horizontal error bars indicate the size of the bins). We then computed the average $E_{p}$ for these 100 points. The most intriguing result is that the correlation appears to be positive for $\alpha<-0.7$ and negative for $\alpha>-0.7$. Performing a Kendell's $\tau$ test on all of the (unbinned) data, we find a $9 \sigma$ positive correlation between $\alpha$ and $E_{p}$ in the $I P D$ regime. To account for both the error in $\alpha$ and $E_{p}$, we have performed this test on all permutations of correlations between the lower and upper values of $\alpha$ (from the $1 \sigma$ error bars) with the lower and upper values of $E_{p}$. In addition we have averaged the value of the correlation statistic $\tau$ from 100 sets of data, in which - for each data point - $\alpha$ and $E_{p}$ are drawn from Gaussian distributions with means equal to the parameter values given in the catalog and standard deviations corresponding to the er- ror bars. In all cases, we find a highly significant $(>6 \sigma)$ correlation. The positive correlation between $\alpha$ and $E_{p}$ in the IPD regime can be simply understood by the instrumental effect discussed in the previous section.

On the other hand, we find a $4 \sigma$ negative correlation between $\alpha$ and $E_{p}$ in the SPD regime. Again, to account for the error in both $\alpha$ and $E_{p}$, we have performed this test on all permutations of correlations between the lower and upper values of $\alpha$ (from the $1 \sigma$ error bars) with the lower and upper values of $E_{p}$, and have also averaged the $\tau$ value from 100 sets of data drawn from distributions based on the existing data, according to the prescription described in the above paragraph. In all of these cases, we find a significant $(>3 \sigma)$ negative correlation. As mentioned above, this type of correlation is natural in the small pitch angle regime, as a result of a decreasing average pitch angle in the electron distribution.

The observed trends are consistent with what is expected from our model in each emission scenario, and tell us something important about the role various effects play in the correlations. For example, the dashed line in Figure 4 shows how $E_{p}$ should change as a function of $\alpha$ in the BATSE spectral window, if only the mean of the electron pitch angle $\Psi$ changes (all other parameters such as $B$ and $\gamma_{m}$ remaining constant). The fact that the observed correlation is weaker could be due to a number of different physical effects. ${ }^{1}$ Of course, we expect that the correlation will be washed out to some degree by dispersion in the intrinsic values of $\Psi$ and $\gamma_{m}$, as well as variation in the magnetic field from burst to burst. It is also possible that the minimum electron Lorentz factor or the magnetic field of the electrons increases as we transition to a physical regime in which electrons are accelerated primarily along the magnetic field lines, which would in turn cause a more gradual decrease of $E_{p}$ with $\Psi$ (or $\alpha$ ). This may be a very plausible explanation - there may exist physical situations which require either a higher magnetic field or characteristic electron Lorentz factor, in which it is very efficient to accelerate along the magnetic field lines. On the other hand, the electrons' Lorentz factors could be only mildly relativistic (instead of $\sim 100$ as we assumed for the dashed line in Figure 4), which would lead to a smaller relative decrease $\left(\sim 1 / \gamma_{e}\right)$ in $E_{p}$ as a function of pitch angle. Finally, it is of course possible that this model is incorrect and an alternative explanation is needed to accommodate bursts above the $\alpha=-2 / 3$ line of death. Still, it is encouraging that the global distribuitons and observed trends are accomodated well by our scenario.

\footnotetext{
1 The positive instrumental correlation discussed in the previous section will also play a small role in reducing the strength of the negative correlation.
} 


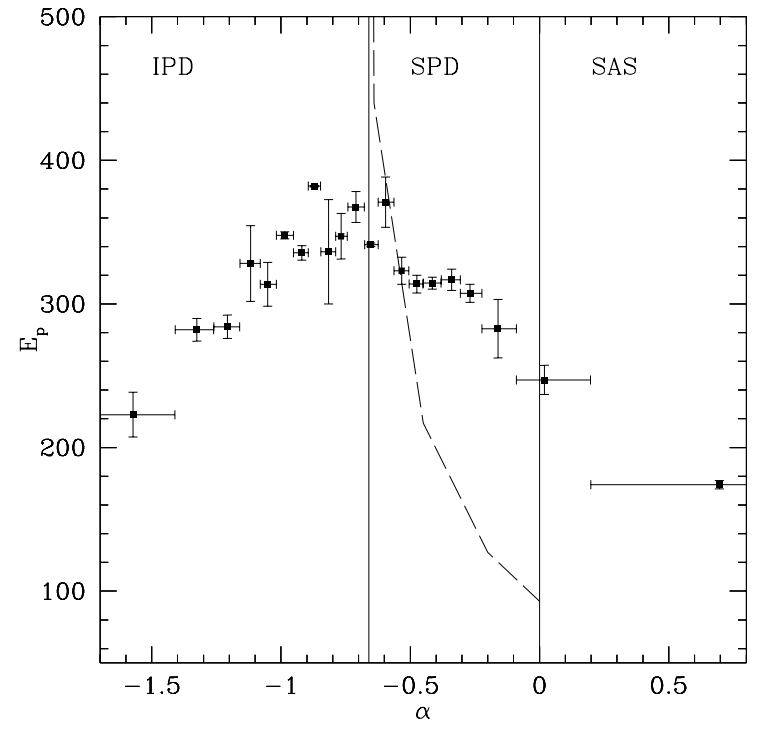

FIGURE 4. Observed $E_{p}$ vs. $\alpha$, for $\sim 2000$ time resolved spectra. Note the change in the sign of the correlation as $\alpha$ transistions from the IPD to SPD regime.

\section{Particle Acceleration and Remaining Questions}

Our results bring to light the fact that particle acceleration in GRBs is a quite poorly understood problem. Usually, it is assumed that the radiating particles in GRBs are accelerated via repeated scatterings across the (internal) shocks. This mechanism, however, predicts several features in the electron distribution not borne out by the data. First, it has been shown [12] that these repeated crossings of the shock result in a power law particle distribution with a well defined index, $p=-2.23$, which would give a high energy synchrotron photon index $\beta$ of -1.62 (or -2.12 for the "cooling" spectrum, e.g. [13]). Although this is consistent with some afterglows, this is certainly is not true for many bursts in the prompt phase. In our synchrotron models above, the high energy photon index $\beta=-(p+1) / 2$, where $p$ is the high energy index of the emitting particle distribution. The parameter $\beta$ can vary by a factor of 4 (or more!) throughout a single burst (see, e.g., [11]), reflecting a huge variation (from 1 to 9 ) in the parameter $p$ of the underlying particle distribution - this is well beyond the statistical limits placed on $p$ by shock acceleration simulations. ${ }^{2}$ In ad-

\footnotetext{
2 Preliminary results indicate that, instead, our adopted picture of stochastic acceleration can produce the necessary electron distributions needed to explain the observed photon spectra in at least the IPD synchrotron emission scenario.
}

dition, shock acceleration predicts an isotropic distribution of electrons. Our work suggests that in a large fraction of GRBs, the particle acceleration is not isotropic but along the magnetic field lines. Thus, there are many crucial open questions related to the physics of particle acceleration in relativistic plasmas, and it is clear that a complete investigation of this phenomenon in the context of GRBs is necessary.

\section{PROMPT X-RAY EMISSION}

Although most GRBs emit the bulk of their prompt radiation in the soft gamma-ray energy band (as their names imply), with the availability of recent X-ray observations of the GRB prompt emission from GINGA [9], BeppoSAX [15, 16], untriggered low energy BATSE data [17], and HETE-II [18], an increasing number of GRBs with spectra that peak in the X-ray energy range $(\sim 1-40$ $\mathrm{keV}$ ) have been discovered. Current estimates [17, 15], suggest that $\sim 30 \%$ of all bursts may have $E_{p}$ values below $\sim 40 \mathrm{keV}$. There has been considerable speculation over the possibility that these low $E_{p}$ spectra may be a result of redshift effects - e.g. that these GRBs occur extremely high redshifts $((1+z)>5)$, and therefore their observed spectra are shifted into the X-ray band. However, as we show below, intrinsic properties of the GRB could also very well produce low peak energies. Below we discuss the role of both cosmological and intrinsic effects in producing X-ray rich GRBs.

\section{Cosmological Effects}

It is straightforward to ask: if we take a burst with a "typical" flux and peak energy at a redshift of 1 and we redshift it until its peak energy falls in the X-ray band, would the burst be detectable (by current instruments)?. Consider a burst at redshift $z$ with specific luminosity $L\left(\mathrm{v}_{o}\right)$ in the cosmological rest frame of the source. The observed flux per unit frequency is

$$
f_{v} \propto \frac{L(v(1+z))}{d \Omega d_{\text {metric }}^{2}(1+z)}
$$

where $v=v_{o} /(1+z), d \Omega$ is the geometric solid angle into which the GRB outflow is confined, $d_{\text {metric }}$ is the metric distance defined as

$$
d_{m e t r i c}=\int_{0}^{z}\left(c / H_{o}\right) \frac{d z}{\sqrt{\Omega_{\Lambda}+\Omega_{m}(1+z)^{3}}} .
$$

For our purposes, we assume a simple standard synchrotron spectrum (in the IPD regime). A burst at a redshift of 1 with $E_{p}=200$ and a flux of $10^{-6} \mathrm{erg} / \mathrm{cm}^{2} / \mathrm{s}$ 


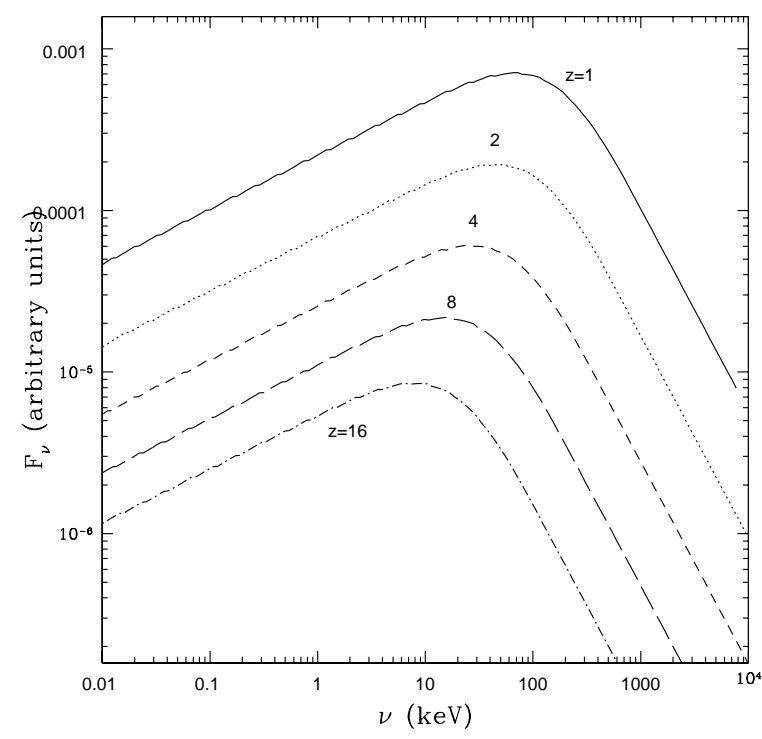

FIGURE 5. A sample $F_{\mathrm{v}}$ spectrum as a function of redshift.

would have an observed flux of about $5 \times 10^{-9}$ when $E_{p}$ is redshifted to $30 \mathrm{keV}$. On the other hand, for a burst with $E_{p}=100 \mathrm{keV}$ and a flux of $10^{-5} \mathrm{erg} / \mathrm{cm}^{2} / \mathrm{s}$ at $z=1$, it is easily detectable (flux $=10^{-8} \mathrm{erg} / \mathrm{cm}^{2} / \mathrm{s}$ ) out to a redshift of 10 , where $E_{p}=10 \mathrm{keV}$. We note that the Heise BeppoSAX sample [15] has fluxes between $10^{-7}$ and $10^{-8}$ $\mathrm{erg} / \mathrm{cm}^{2} / \mathrm{s}$, while the fluxes from Kippen's BATSE sample $[17,23]$ ranged from $10^{-7}$ to $5 \times 10^{-9} \mathrm{erg} / \mathrm{cm}^{2} / \mathrm{s}$. Unfortunately, because of the broad GRB luminosity function and intrinsic $E_{p}$ distribution (i.e. because there are no standard intrinsic luminosity and $E_{p}$ values for all GRBs) all we can say at this point is that it is possible that these low $E_{p}$ bursts are very high redshift bursts. We do note that the highest fluxes in both data samples require the bursts to be on either the high end of the intrinsic luminosity distribution or the low end of the intrinsic $E_{p}$ distribution in order to be consistent with the redshift interpretation. Nonetheless, we can neither rule out nor strongly favor the high redshift interpretation of these bursts.

\section{Luminosity Evolution}

There have been some suggestions that GRB luminosity function evolves with redshift, in the sense that bursts at high redshift tend to have intrinsically higher luminosity [19]. This statement is based on those bursts with redshifts and luminosities from the luminosity-variability relation [20,21], and so caution should be exercised until this relation is more definititively shown to provide valid redshifts. However, if we do adopt the redshifts

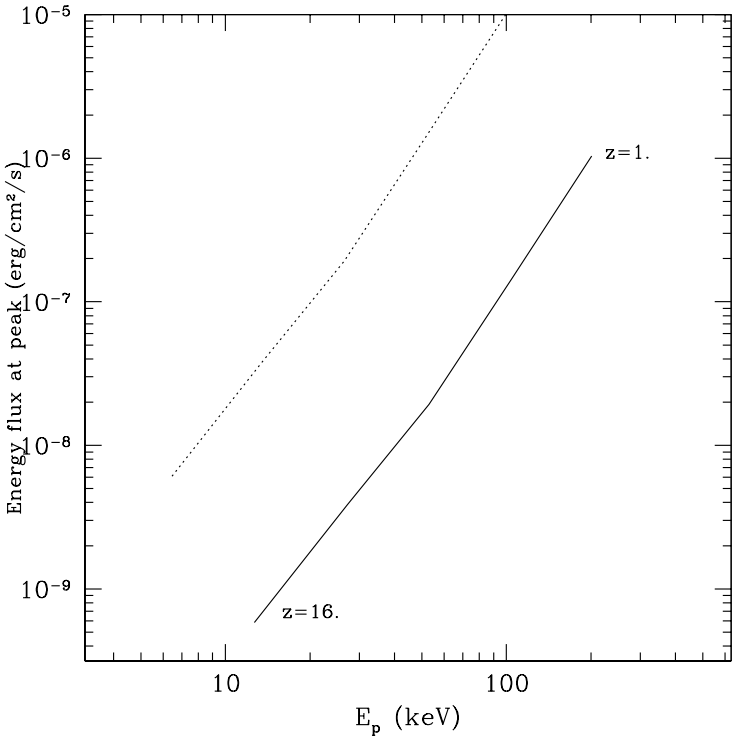

FIGURE 6. Energy flux at the peak of the $F_{\mathrm{v}}$ spectrum vs. $E_{p}$ for changing redshifts of the burst, given two initial "starting" values of $E_{p}$ and flux at $z=1$.

and luminosities from the L-V relation, then there is significant evidence that GRBs are brighter at higher redshifts. This effect allows for an even greater probability that very high redshift bursts are detectable by current instruments. For example, [19] find the average GRB luminosity $L \sim(1+z)^{1.4 \pm 0.5}$. Therefore, the observed flux decreases less rapidly as a function of redshift, than if there were no luminosity evolution. ${ }^{3}$

\section{Intrinsic Effects}

The analysis above considered how a particular burst behaves as it is moved out in redshift space, for its particular set of observed properties. However, as has been suggested repeatedly and confirmed by bursts with measured redshifts, GRB intrinsic properties vary substantially from burst to burst. In some cases, the intrinsic properties can act in such a way as to mimic redshift effects.

For example, again assuming a standard synchrotron spectrum (which we do to be concrete, but which is not necessary), we can investigate how the $v F_{v}$ flux changes

\footnotetext{
3 However, we mention one important caveat: If whatever causes the GRB luminosity function to evolve also causes $E_{p}$ to evolve, then there would be a decreased dependence of $E_{p}$ on redshift and one would have to place the burst at even higher redshifts to shift $E_{p}$ into the X-ray band.
} 


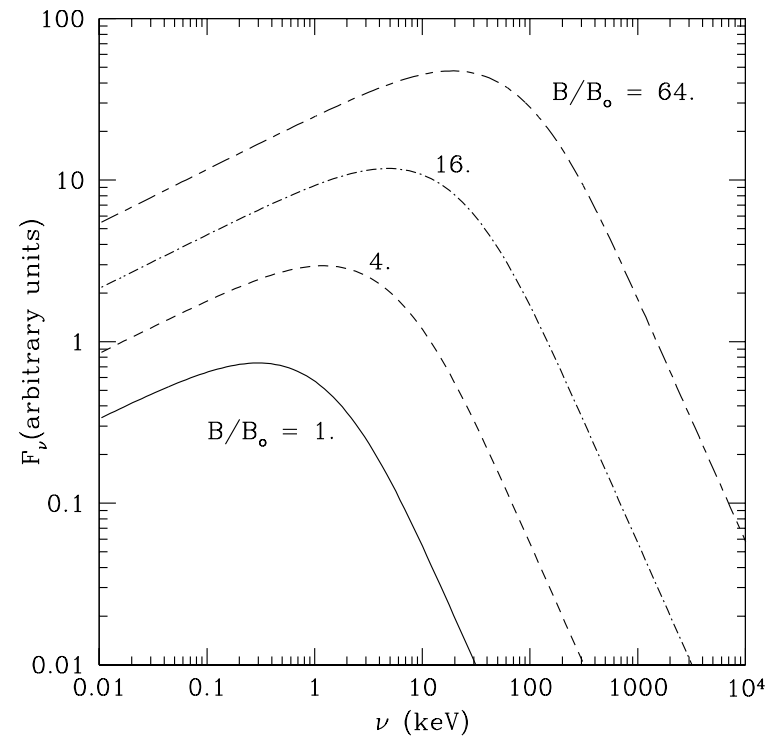

FIGURE 7. A synchrotron $F_{v}$ spectrum as a function of magnetic field.

as a function of various physical parameters. We take the magnetic field as an example. Figure 7 shows how the spectrum shifts with decreasing magnetic field from the $\gamma$-ray to X-ray regime. The flux at the peak is approximately proportional to $B^{2}$ while the peak energy goes as $B$. Figure 8 shows the peak flux as a function of $E_{p}$. Here we see that changing the magnetic field could also very reasonably produce $\mathrm{X}$-ray bursts. Other possibilities include decreasing the bulk Lorentz factor, or changing the electron spectrum (such as the density or minimum energy of the electrons).

There are in fact several pieces of evidence suggesting that intrinsic effects are playing the dominant role in producing the low $E_{p}$ bursts:

1. One clue comes from the fact that the Kippen et al. bursts [17, 23], fall along an extension of the well known observed hardness-intensity correlation [24] in BATSE GRBs (see Figure 3 of [23]). Lloyd, Petrosian, \& Mallozzi [22] found that this correlation cannot be produced by redshift effects alone, given any reasonable (i.e. not a $\delta$-function) GRB luminosity function. That is, even for a fairly narrow intrinsic peak energy distribution, the observed hardness-intensity correlation cannot be due to cosmological expansion (e.g. lower flux and lower $E_{p}$ do not necessarily imply higher redshifts because the broadness of the GRB luminosity function washes out this effect). In fact, they found that a correlation between the intrinsic peak energy and the emitted energy of the GRB would naturally reproduce the observed correlation, and that such a relation between peak energy and total energy/luminosity is natural in (but not limited to) a syn-

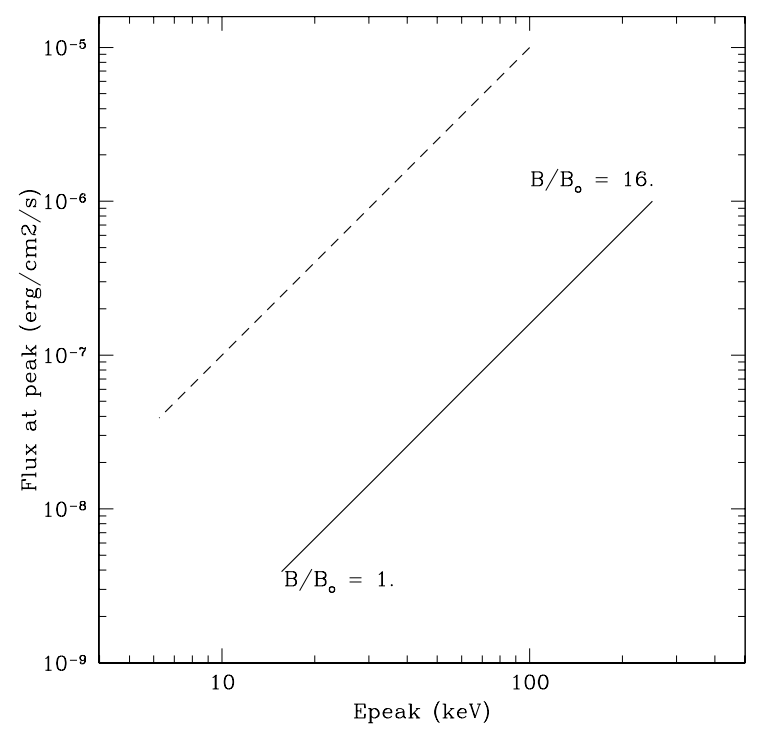

FIGURE 8. Same as Figure 6, but for a changing magnetic field.

chrotron emission model.

2. The observed durations of the XRBs are not unusually long or smooth. At the highest redshifts (e.g. $1+z \sim 10$ ), the duration and pulse width are dilated by a factor of 10. Although there exists no standard in GRB temporal properties, one might expect that if these bursts were at very high redshifts, their time profiles would be particularly long or smooth - this does not seem to be the case. Heise et al. [15] also show that the durations of the bursts in his sample are completely consistent with the global population and not unusually long.

3. The Kippen and Heise sample of low $E_{p}$ bursts are based on the average (over the duration of the burst) spectral properties of the burst. However, there have been some time resolved spectral analyses in which observations of X-ray rich pulses within a burst are reported (e.g. $[25,16])$. For at least these bursts, the X-ray "richness" of the pulses must therefore be coming entirely from intrinsic effects.

\section{PROMPT OPTICAL AND VERY HIGH ENERGY EMISSION}

Optical There are several mechanisms in a GRB that may produce prompt emission at optical wavelengths. And in fact in one burst - GRB 990123 - such emission was seen as a bright optical flash $\left(m_{v} \sim 9\right)$ during the prompt phase [26]. The most common interpretation of this optical flash is that it resulted from the blast wave reverse shock propagating into the dense GRB ejecta. 
Because the temperature in this region is smaller relative to the shocked ambient medium by a factor of $\sim \Gamma^{2}$, the emission from this interaction peaks in the optical energy band. Certain conditions (sensitive to $\Gamma$ ) are required to actually produce such a flash (see [27, 28] for discussion); if this interpretation is correct, it offers the possibility of constraining - among other things - the bulk Lorentz factor and baryonic content of the GRB outflow.

An alternative interpretation of this bright optical flash involves the interaction of the GRB radiation front [29, 30, 31] with the ambient medium. In this scenario, the $\gamma$-ray photons travelling ahead of the blast wave are side-scattered by the ambient medium. These photons then pair produce with the forward streaming photons, and pair enrich the medium. The available energy must distributed among these pairs, and therefore the peak of the initial afterglow emission is lower in energy (relative to the standard picture without pairs) by a factor of $\left(m_{e} / m_{p}\right)^{2}$. As a result, the very early GRB afterglow (possibly coincident with the prompt emission) is brightest in the optical. In order for this mechanism to work efficiently, the medium is constrained to a particular density profile (see [29] for more details). Hence, if this mechanism succeeds, the prompt optical GRB emission affords us the opportunity to constrain the surrounding GRB environment.

Very High Energy If synchrotron emission is indeed responsible for the prompt GRB emission in the low energy gamma-ray band (see above), then we expect an inverse-Compton component from this emission. The inverse Compton component is boosted by a factor of $\Gamma^{2}$ and therefore peaks in the $\sim 10 \mathrm{GeV}$ range. There is also the possibility of observing very high (i.e. TeV) emission from a high energy proton component [32], as well as other high energy phenomena (such as neutrinos; see [33] for a discussion). A number of upcoming instruments (GLAST, Agile, Milagro, etc) will be sensitive in the range from 10's of MeV to TeV energies and have the potential of shedding much light on this relatively unexplored but important energy range of of GRB emission.

\section{CONCLUSIONS}

We have discussed the prompt emission of GRBs, focusing particularly on the gamma-ray and X-ray emission properties. We find that a generalized synchrotron emission model does a good job of explaining the behavior of the prompt spectra in the $\sim 20 \mathrm{keV}-1 \mathrm{MeV}$ range. Moreover, we find that although the increasing number of low $E_{p}(<40 \mathrm{keV})$ GRBs may be consistent with a high redshift interpretation, the data appear to suggest that in fact variations in the bursts' intrinsic properties (e.g. magnetic field, electron energy density, etc.) are probably responsible for producing these "X-ray rich" bursts. We emphasize the importance of broadband (from $\mathrm{eV}$ to $\mathrm{TeV}$ ) observations of prompt emission in order to gain a complete understanding of the physics of GammaRay Bursts.

\section{ACKNOWLEDGMENTS}

I would like to thank the organizers for an interesting and stimulating conference. I would also like to thank Vahe' Petrosian, with whom much of the work on the prompt gamma-ray emission was done.

\section{REFERENCES}

1. Lloyd, N.M. \& Petrosian, V. 2000, ApJ, 543, 722

2. Lloyd-Ronning, N.M. \& Petrosian, V. 2001, ApJ, in press

3. Band, D., et al. 1993, ApJ, 413, 281

4. Tavani, M. 1996, ApJ, 466, 768

5. Preece, R.D., et al. 1996, ApJ, 473, 310

6. Ghissellini, G., Celotti, A., Lazzati, D. 2000, MNRAS 313, L1

7. Katz, J. 1994, ApJ, 432, L107

8. Epstein, R.I. 1973, ApJ, 183, 593

9. Strohmeyer, T.E., et al. 1998, ApJ, 500, 873

10. Dung, R. \& Petrosian, V. 1994, ApJ, 421, 550

11. Preece, R.D., et al. 1999, ApJS, 126, 19

12. Kirk, J.G. et al., 2000, ApJ, 542, 235

13. Sari, R., Piran, T., \& Narayan, R. 1998, ApJ, 497, L17

14. Piran, T. 1999, Physics Reports, 314, 575

15. Heise, J. et al. 2001, these proceedings.

16. Frontera, F., et al. 2000, ApJS, 127, 59

17. Kippen, R. M., et al. 2001, these proceedings

18. Barraud, C., et al. 2001, these proceedings

19. Lloyd-Ronning, N. M., Fryer, C. L., \& Ramirez-Ruiz, E. 2001, ApJ, submitted (astro-ph/0108200)

20. Fenimore, E. E., \& Ramirez-Ruiz, E. 2001, ApJ, submitted (astro-ph/0004176)

21. Reichart, D. E., et al. 2001, ApJ, 552, 57

22. Lloyd, N.M. et al., 2000, ApJ, 534, 227

23. Kippen, M. et al., 2001 in "Gamma-Ray Bursts in the Afterglow Era", Rome, in press.

24. Mallozzi, R.S. et al. 1996, in Gamma-Ray Bursts, AIP Conf. Proc. 384, eds. C. Kouveliotou, M.F. Briggs, G.J. Fishman (New York: AIP), 204.

25. Smith, D.A., et al. 2001, ApJ, submitted

26. Akerlof, C., et al., 1999 Nature 398, 400

27. Sari, R. \& Piran, T. 1999, ApJ, 517, L109

28. Soderberg, A. \& Ramirez-Ruiz, E., these proceedings

29. Beloborodov, A.M., 2001, ApJ, in press

30. Thompson, C. \& Madau, P. 2000, ApJ, 538, 105

31. Meszaros, P., Ramirez-Ruiz, E., Rees, M.J. 2001, ApJ, 554,660

32. Totani, T., 1999, MNRAS, 307, L41

33. Waxman, E. 2000, ApJS, 127, 519 